\title{
High-fat diet accelerates progression of osteoarthritis after meniscal/ligamentous injury
}

\author{
Robert A Mooney ${ }^{1,2^{*}}$, Erik R Sampson², Jaclyn Lerea ${ }^{1}$, Randy N Rosier ${ }^{2}$ and Michael J Zuscik ${ }^{2}$
}

\begin{abstract}
Introduction: Increasing obesity and type 2 diabetes, in part due to the high-fat (HF) Western diet, parallels an increased incidence of osteoarthritis (OA). This study was undertaken to establish a causal relation between the HF diet and accelerated OA progression in a mouse model and to determine the relative roles of weight gain and metabolic dysregulation in this progression.
\end{abstract}

Methods: Five-week-old C57BL/6 mice were placed on HF (60\% kcal) or low-fat (lean, 10\% kcal) diets for 8 or 12 weeks before transecting the medial collateral ligament and excising a segment of the medial meniscus of the knee to initiate OA. One group was switched from lean to HF diet at the time of surgery.

Results: Body weight of mice on the HF diet peaked at $45.9 \pm 2.1 \mathrm{~g}$ compared with $29.9 \pm 1.8 \mathrm{~g}$ for lean diets, with only those on the HF becoming diabetic. Severity of OA was greater in HF mice, evidenced by the Osteoarthritis Research Society International (OARSI) histopathology initiative scoring method for mice and articular cartilage thickness and area. To assess the importance of weight gain, short- and long-term HF diets were compared with the lean diet. Short- and long-term HF groups outweighed lean controls by $6.2 \mathrm{~g}$ and $20.5 \mathrm{~g}$, respectively. Both HF groups became diabetic, and OA progression, evidenced by increased OARSI score, decreased cartilage thickness, and increased osteophyte diameter, was comparably accelerated relative to those of lean controls.

Conclusions: These results demonstrate that the HF diet accelerates progression of OA in a type 2 diabetic mouse model without correlation to weight gain, suggesting that metabolic dysregulation is a comorbid factor in OArelated cartilage degeneration.

\section{Introduction}

Decreased physical activity and the consumption of a high-fat Western diet have contributed to a worldwide epidemic of obesity and associated type 2 diabetes [1]. As a result, the incidence of cardiovascular disease, hypertension, cancer, nonalcoholic fatty liver disease, stroke, and other secondary complications is anticipated to be negatively affected [2,3]. One additional consequence of this epidemic is an increased incidence of osteoarthritis (OA) in both weight-bearing and nonweight-bearing joints $[4,5]$, with more than $50 \%$ of those with diabetes having some form of arthritis [4]. In the United States, $52.1 \%$ of patients receiving knee replacements in 2005 were obese [6]. In Canada, 87\% were

\footnotetext{
*Correspondence: Robert_Mooney@urmc.rochester.edu 'Department of Pathology and Laboratory Medicine, Box 626, University of Rochester Medical Center, 601 Elmwood Avenue, Rochester, NY 14642, USA Full list of author information is available at the end of the article
}

either obese or overweight [7]. A morbidly obese patient is 33 times more likely to require knee replacement than is an individual of normal body mass [7]. Despite these statistics, current knowledge does not adequately explain the relative contribution to OA of biomechanical changes resulting from increased body mass versus the metabolic dysfunction in obesity. Although it is widely accepted that the increased biomechanical stresses on the weight-bearing diarthrodial joints due to obesity can initiate and accelerate the OA disease process [8], the potential influence of metabolic dysfunction on the progression of $\mathrm{OA}$ is receiving increasing attention $[9,10]$.

Increased dietary fat, as consumed in Western diets, can contribute to both obesity and the metabolic dysfunction that is associated with insulin resistance/type 2 diabetes. Surplus dietary fat is deposited in adipose tissue as well as in skeletal muscle, heart, and liver. As this fat burden increases, adverse effects on systemic

\section{() Biomed Central}


metabolism increase [11]. The current model of insulin resistance of obesity as a proinflammatory state is based in part on the observation that adipose tissue in obese individuals and animal models contains increased numbers of activated macrophages that release proinflammatory cytokines such as interleukin-1, interleukin-6, and tumor necrosis factor- $\alpha[12,13]$. These cytokines act both locally on adipocytes and systemically to impair insulin action in insulin-target tissues $[11,14,15]$. Metabolism of the abundant circulating free fatty acids in the high-fat diet can also lead to oxidative stress in cells, formation of lipid peroxides, and subsequent oxidative damage to DNA, RNA, and proteins [16-18]. Oxidative stress has been more specifically shown to inhibit insulin-receptor signaling pathways in cells, at least in part through activation of the stress kinases JNK and p38 and to impair mitochondrial function $[19,20]$. The net effect of the proinflammatory state and tissue oxidative stress is a systemic metabolic dysfunction that defines the metabolic syndrome and type 2 diabetes that are associated with high-fat diets and obesity.

Although it is widely accepted that the increased biomechanical stress associated with obesity contributes to the initiation/progression of OA [21-23], the importance of type 2 diabetes-related systemic metabolic dysregulation in the OA disease process has not been delineated. To begin to address this question, an animal model was used to test the hypothesis that HF diet-induced obesity and associated glucose intolerance/insulin resistance accelerate the progression of osteoarthritis after meniscal/ligamentous injury (MLI). This model was subsequently used to determine whether marked obesity is required for the HF diet to accelerate OA.

\section{Materials and methods \\ Animals}

Male C57BL/6J mice purchased from Jackson Laboratories (Bar Harbor, ME) were housed five per cage in a microisolator room on a 12-hour light/dark cycle at the University of Rochester. Mice were placed on a high-fat (60\% kcal, D12492) or low-fat (10\% kcal, D12450B) diet at 5 weeks of age (Open Source Diets, Research Diets Inc., New Brunswick, NJ). After 8 or 12 weeks on the diet, mice were anesthetized by using intraperitoneal (IP) injection of $60 \mathrm{mg} / \mathrm{kg}$ ketamine and $4 \mathrm{mg} / \mathrm{kg}$ xylazine, and the medial collateral ligament of the right hindlimb was transected, and a segment of the medial meniscus detached and excised by using a surgical method [24] that we have established in our laboratory [25]. It should be noted that this model of injury, termed MLI, leads to posttraumatic OA that is detectable by 4 weeks after injury and progresses over a 4month period, similar to that seen in the DMM model of posttraumatic OA [26]. The contralateral limb was sham-operated and represented the experimental control. Pre- and postsurgery mice were provided analgesia (intraperitoneal (IP) injection of $0.5 \mathrm{mg} / \mathrm{kg}$ buprenorphine) every 12 hours for 72 hours. Before killing mice at monthly intervals, the mice were fasted overnight, weighed, and tail-vein blood samples were taken for blood glucose measurements by using One Touch glucose meters (Lifescan, Inc., Milpitas, CA). Hemoglobin A1c measurements were made on tail-vein blood samples by using a Bayer DCA $2000 \mathrm{HgbA} 1 \mathrm{C}$ analyzer (Deerfield, IL). The assay is based on monoclonal antibody detection of the glycated amino terminus of the $\beta$ chain of human hemoglobin. The University Committee on Animal Resources approved all protocols.

\section{Tissue harvest and histologic analysis of OA progression}

After establishment of deep anesthesia by using IP injection of $60 \mathrm{mg} / \mathrm{kg}$ ketamine and $4 \mathrm{mg} / \mathrm{kg}$ xylazine, mice were killed via cardiac perfusion by using $10 \mathrm{ml}$ of phosphate-buffered saline followed by $10 \%$ neutral buffered formalin. Isolated knee joints were fixed for 3 days in $10 \%$ neutral buffered formalin at $23^{\circ} \mathrm{C}$. Samples were decalcified for 2 weeks in 10\% wt/vol EDTA and embedded in paraffin. Three-micrometer-thick sagittal sections from the medial joint compartment of the right knee were cut on a microtome and mounted on positively charged slides, baked at $60^{\circ} \mathrm{C}$ for 30 minutes, deparaffinized in xylene, and rehydrated in decreasing concentrations of ethanol.

Mouse knee-joint sections were stained with both Safranin O-fast green and Alcian blue-hematoxylin to allow visualization of cartilage tissue. Semiquantitative histopathologic grading of Alcian blue-stained knee-joint cartilage was performed on sagittal sections by using a murine scoring system established by the OARSI histopathology initiative [27], in which $0=$ normal cartilage, $0.5=$ loss of proteoglycan stain without cartilage damage, 1 = mild superficial fibrillation, 2 = fibrillation and/or clefting extending below the superficial zone, $3=$ mild $(<25 \%)$ loss of cartilage, $4=$ moderate $(25 \%$ to $50 \%)$ loss of cartilage, $5=$ severe $(50 \%$ to $75 \%)$ loss of noncalcified cartilage, and $6=$ eburnation with $>75 \%$ loss of cartilage.

\section{Quantification of cartilage morphologic features}

After histologic grading, Alcian blue-stained sagittal sections were evaluated for cartilage area and thickness by using OsteoMeasure software (OsteoMetrics, Decatur, GA), as previously described [28]. Articular cartilage was defined as the area from the joint surface to the junction of the deep zone of the cartilage with the subchondral bone, including the entire surface from the anterior to the posterior edges of both the tibia and the femur. Cartilage area was quantified in each section by 
using an area-calculating algorithm in the software, as we previously described [25]. Similarly, tibial cartilage thickness was analyzed at the center of the tibial plateau. Osteophytes were positively identified by using the following criteria: the osteophyte (a) protruded into the joint space, (b) contained hypertrophic chondrocytes, and (c) displayed evidence of calcification. Histologic assessment of osteophytes was performed on each slide by using a digital projection at $\times 100$ magnification. The largest cross-sectional dimension of the osteophyte was measured, as was the perpendicular dimension. These two determinations were averaged. Where multiple osteophytes were detected, the mean of the average osteophyte diameters was recorded. All three parameters (thickness, area, osteophyte size) were determined and averaged among all sections. Each diet group contained five to eight mice, with three sections from each joint being analyzed in the histologic grading and histomorphometric analyses.

\section{Micro-computed tomography (micro-CT) evaluation of mouse knee joints}

Before histologic processing, knee joints were evaluated with micro-CT by using a Scanco vivaCT 40 scanner with 55-kVp source (Scanco USA, Inc., Wayne, PA). Joints were scanned at a resolution of $12 \mu \mathrm{m}$, with a slice increment of $10 \mu \mathrm{m}$. Images from each group were reconstructed at identical thresholds to allow 3-dimensional structural rendering of each joint. Analysis of bone volume was performed on selected regions between the femoral and tibial growth plates.

\section{Statistical analysis}

Results are expressed as mean values \pm SEM. Statistical analysis was performed by using StatView 5 software (SAS Institute, Cary, NC). Data were tested for normality by using the Kolmogorov-Smirnov test (with DallalWilkinson-Lilliefor $P$ value). Experimental means were compared by using ANOVA; sample means from three or more groups were compared, and the Fisher protected least significant differences test was used for comparisons between pairs of groups. A calculated $P$ value of less than 0.05 was considered significant when comparing between-group differences.

\section{Results}

Five-week-old C57BL/6 mice were placed on either an HF diet representing $60 \%$ of total dietary kcal or an LF (lean) diet with $10 \%$ of kilocalories derived from fat. The HF diet has been shown to cause hyperglycemia and insulin resistance in this strain of mice [29]. After 8 weeks, mice on the HF diet weighed $29 \%$ more than those on the lean diet $(30.9 \pm 0.9$ g versus $23.9 \pm 0.6 \mathrm{~g}$; $P<0.0001$; Figure 1a). Fasting blood glucose levels were in the diabetic range only for the HF group (182.3 \pm $10.5 \mathrm{mg} / \mathrm{dl}$; Figure 1B), confirming a dysmetabolic state associated with the HF diet.

Progression to severe OA is typically observed within 5 months by using the meniscal-ligamentous injury (MLI) technique [25]. With the objective to determine whether the HF diet with its associated type 2 diabetic phenotype accelerates OA progression, mice from each dietary group ( $n=4$ to 9 ) were killed at monthly intervals out to 4 months after MLI surgery. Both groups gained weight over this 4-month period, with the HF group becoming markedly obese, with a peak weight of $45.9 \pm 2.1 \mathrm{~g}$ compared with only $29.9 \pm 1.8 \mathrm{~g}(P<$ 0.0001 ) for the lean group (Figure 1a). As expected, the HF group was diabetic (fasting blood glucose greater than $125 \mathrm{mg} / \mathrm{dl}$ ), with peak levels of $288.8 \pm 21.4 \mathrm{mg} / \mathrm{dl}$ at 4 months (Figure 1b). Histologic analysis of the articular cartilage at the 4-month time point revealed increased fibrillation, clefting, and decreased proteoglycan staining in response to MLI. These changes were more pronounced in the HF group $(P<0.01$; Figure 2a) with major loss of cartilage. In some cases, this loss progressed nearly to the subchondral bone. No significant cartilage loss was observed in sham-operated knees of either HF or lean groups, with only occasional superficial zone fibrillation.

A progressive worsening of OA from 2 to 4 months was demonstrated in both diet groups, with the score for OA severity being significantly higher for the HF group at each of these time points (Figure 2b). Data for lean mice at month 1 indicate an unexpectedly advanced stage of OA for that time point. The explanation for this aberrant data point is not clear. With the OARSI score, the HF group on average had $25 \%$ to $50 \%$ loss of articular cartilage at 2 months (score of 4 ), whereas the lean group had less than $25 \%$ loss of articular cartilage at this same time point (score of $3 ; P<0.01$ ). As expected, the sham controls had scores of 1 or less, representing only occasional superficial zone fibrillation, even at 4 months (data not shown).

Histomorphometric analysis of the articular cartilage in the lean and HF groups over the 4-month time course confirmed the accelerated loss of articular cartilage in the HF diet group. Articular cartilage thickness on the tibial plateau progressively decreased in both groups but was approximately $40 \%$ to $50 \%$ thinner in the HF group between 2 and 4 months after MLI (Figure 2c). Similarly, total articular cartilage area, representing the combined cartilage areas of the femur and tibia, decreased progressively with the most marked difference between lean and HF diets being observed at the 4-month time point (Figure 2d). At this time point, cartilage area in the HF group had decreased by an additional $35 \%$ relative to lean controls $(P<0.01)$. 

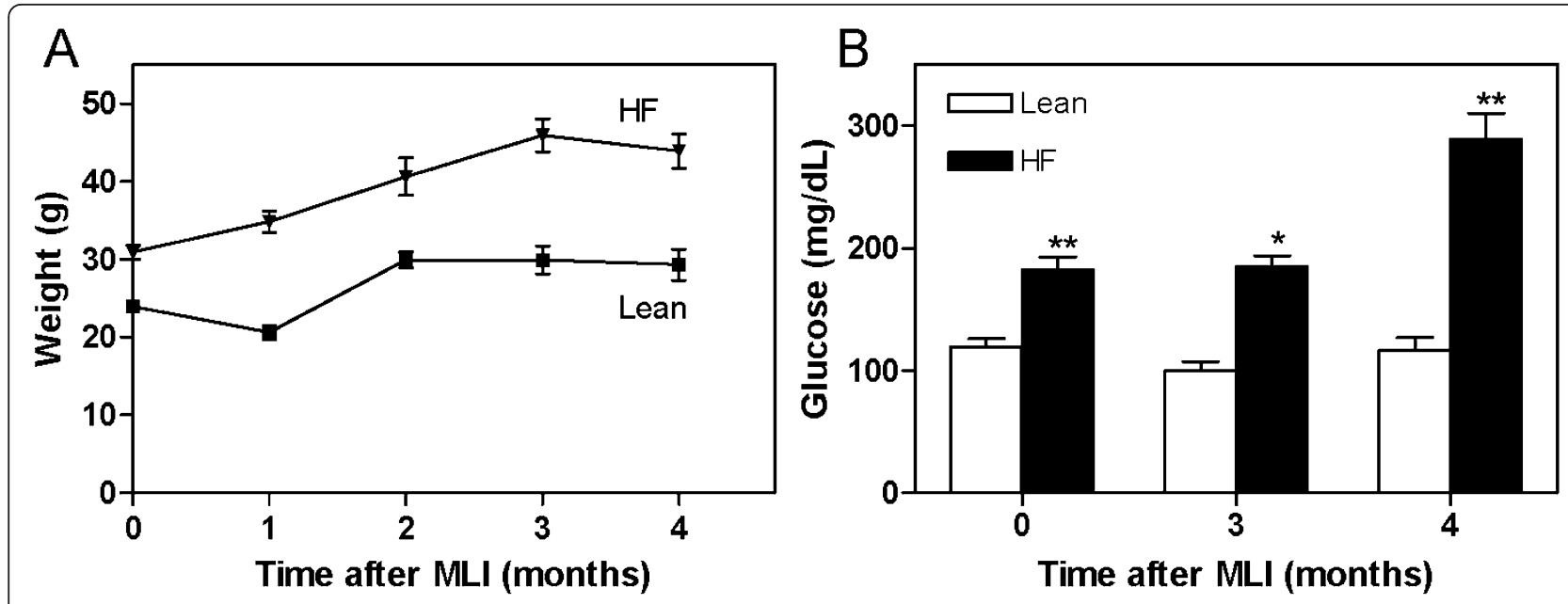

Figure 1 High-fat diet promotes weight gain and hyperglycemia. Five-week-old male C57BL/6 mice were placed on either a high-fat (60\% kcal) or low-fat (10\% kcal) diet for 2 months before meniscal-ligamentous injury (MLI). Body weight (a) and blood glucose levels (b) were determined at monthly intervals after MLI. ${ }^{*} P<0.05 ;{ }^{*} P<0.01 ; n=5$ to 9 mice at each time point.

In the preceding experiments, the HF-fed mice at the later time points were markedly obese in consequence of a 6-month diet protocol that included 2 months of the HF diet before the initiation of OA with MLI. To determine whether this marked increase in body weight was primarily responsible for the accelerated progression of OA with HF feeding, two HF diet protocols were compared. One group of mice $(n=6)$ was fed an HF

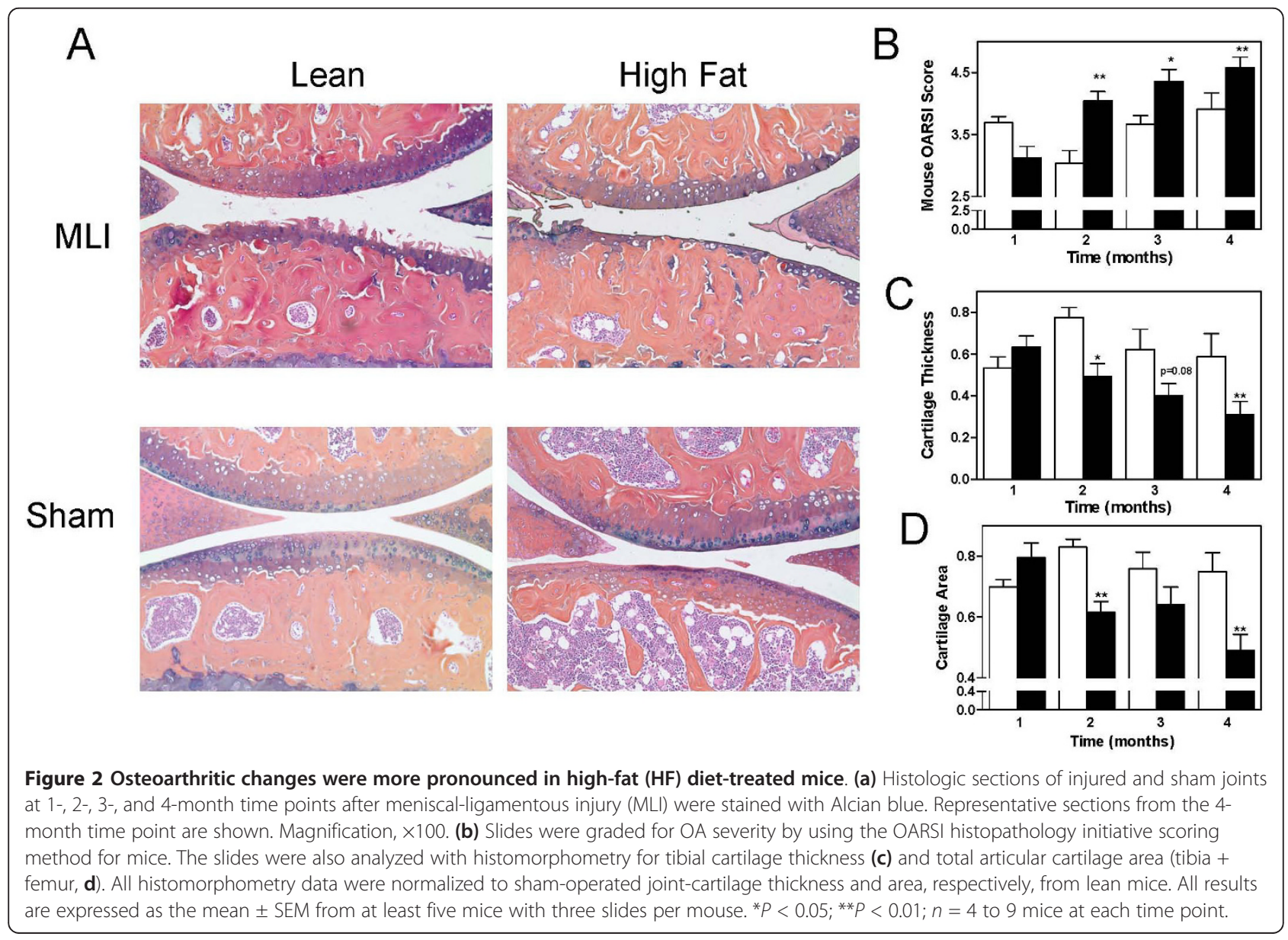


diet for 3 months before MLI to establish obesity before initiation of OA. In a second group $(n=8)$, an HF diet was begun only at the initiation of OA. As a result of beginning the HF diet at the time of MLI, this group of mice demonstrated only a modest increase in body weight relative to the lean diet controls $(n=7)$ (Figure 3a). This contrasts with the long-term HF diet group, which again attained a marked obese body weight that was $80 \%$ above that of lean mouse controls. Despite the modest weight gain, the short-term HF group displayed metabolic dysfunction, as indicated by fasting hyperglycemia at 1-, 2-, and 3-month time points, although levels were less than those of the long-term HF group. Values at 3 months are shown (Figure 3b). An additional marker for a dysmetabolic state, hemoglobin A1c, a measure of average blood glucose levels over the preceding 3-month period [30], was nearly equally elevated in the two HF groups (Figure 3c). Note that the absolute hemoglobin A1c values were less than those of humans, for whom this immunoassay-based test is designed. These results indicate that the short-term HF diet produced metabolic dysfunction without requiring marked obesity or the extended 3-month period on the HF diet before the MLI surgery.

Histologic analysis of the progression of OA at 3 months after MLI confirmed that a long-term HF diet was again associated with accelerated OA progression, with major loss of cartilage relative to the lean controls. In some cases, this loss progressed nearly to the subchondral bone. Importantly, the short-term HF diet group had an equally accelerated progression of OA when compared with the long-term HF group (Figure 4a). No significant cartilage loss was observed in shamoperated knees of either HF or lean groups, with only occasional superficial zone fibrillation. These observations were confirmed by comparably higher
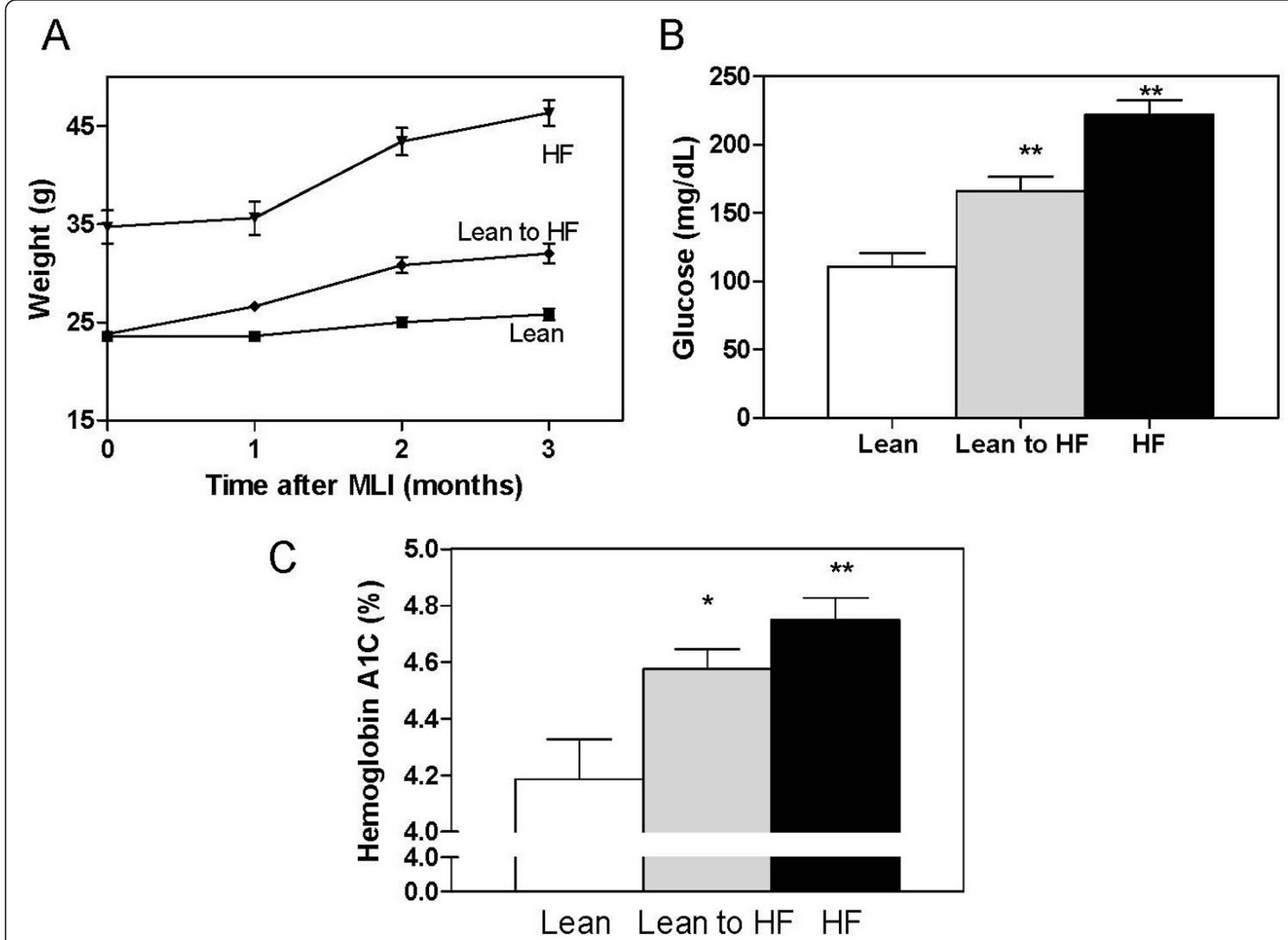

Figure 3 Differing weight gain but similar hyperglycemia in short- and long-term high-fat (HF) diets. Five-week-old male C57BL/6 mice were given either a high-fat ( $60 \% \mathrm{kcal})$ or low-fat (10\% kcal) diet for 3 months before meniscal-ligamentous injury (MLI). After MLI, half of the lean group was switched to the HF diet. The three groups were maintained on diets for an additional 3 months. (a) Body weight was measured monthly after the MLI procedure. (b) Blood glucose and (c) hemoglobin A1c levels were determined at the 3-month end point. Data points represent mean \pm SEM of at least six mice. ${ }^{*} P<0.05$; ${ }^{* *} P<0.01 . n=6$ to 8 mice in each group. 
osteoarthritis grades for the HF groups relative to the lean controls, as assessed by the OARSI scoring method $(P<0.05$; Figure $4 \mathrm{~b})$. It should be noted that the accelerated cartilage degeneration occurred in the short-term HF group despite the absence of marked obesity. In agreement with the OARSI scoring and supporting the idea that marked obesity is not required for acceleration of cartilage degeneration in mice fed an HF diet, tibial plateau articular cartilage thicknesses were $25 \%$ to $50 \%$ lower in the two HF groups compared with the lean group (Figure 4c). Cartilage-area determination supported the thickness data by demonstrating similar trends toward decreased area in both HF diet groups relative to mice fed the lean diet (data not shown). These results indicate that accelerated progression of $\mathrm{OA}$ in mice on an HF diet is associated with a diabetic metabolic phenotype, does not correlate with degree of weight gain, and does not require prolonged exposure to the HF diet.

Quantitative micro-CT analysis of knee joints at the 3month time point showed increased bone volume in the MLI knees relative to sham-treated controls, consistent with OA-like subchondral sclerosis and joint/periarticular mineralization. Only differences in the HF-diet

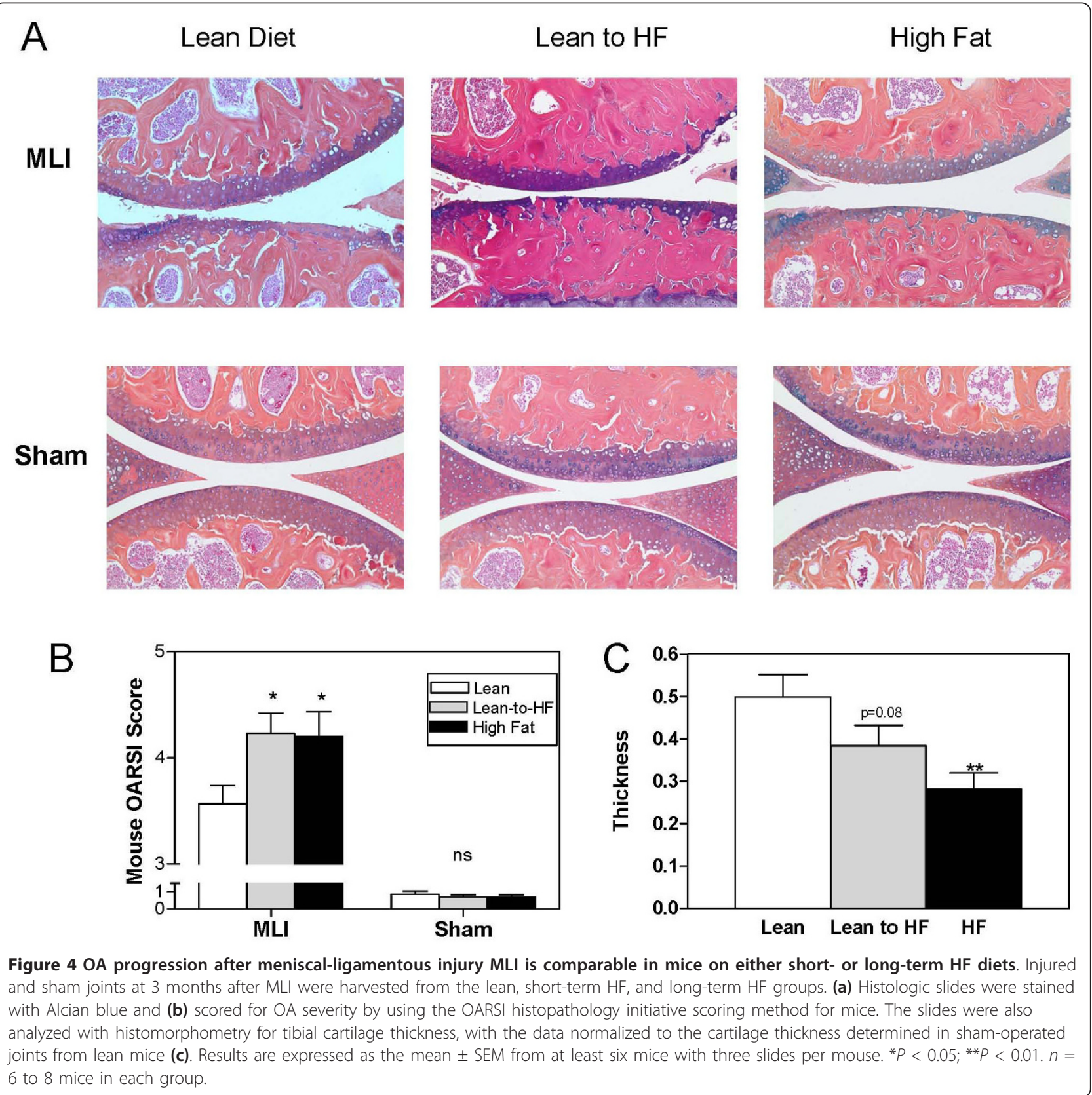


groups, however, were statistically significant $(P<0.01$; Figure 5a).

Regarding enhanced joint and periarticular mineralization specifically, 3-dimensional reconstructions of the raw micro-CT data revealed a more progressive meniscal calcification and a suggestion of increased presence of osteophytes in both high-HF groups relative to the lean diet controls (Figure 5b). To investigate the possibility that HF diets promote the formation of osteophytes, all histologic slides were evaluated for the presence and size of osteophytes on the tibial surface. The blinded analysis revealed osteophytes in essentially all MLIinjured knees from all diet groups (Figure 6a). Importantly, not only was the size of the osteophytes greater in both HF-diet groups $(P<0.01)$, but they also were of comparable size in the short-term and long-term HF groups (Figure 6b). Interestingly, 30\% of sham knees from HF-diet mice contained small osteophytes (Figure $6 c$ ), and another $20 \%$ showed early changes suggestive of osteophytes. This was distinct from sham knees from the lean mice, which did not display osteophytes, with early changes evident only in less than $20 \%$ of knees (Figure 6d). As with progression of cartilage

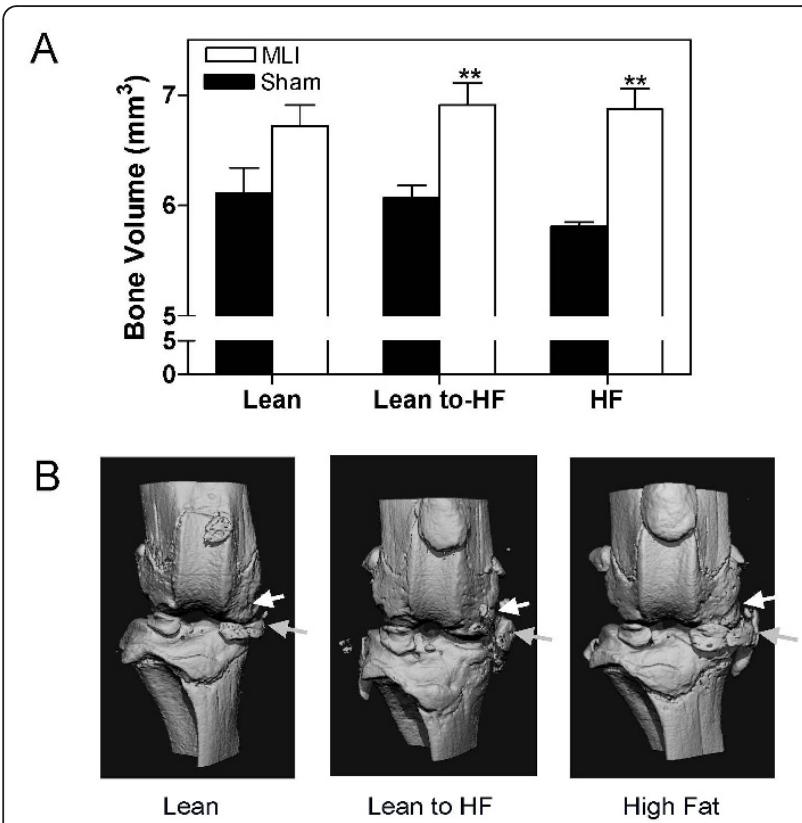

Figure 5 Micro-CT analysis of knee joints after meniscalligamentous injury (MLI) as a function of diet. (a) Knee-bone volumes of MLI and sham-treated limbs at 3 months in HF, Lean to $H F$, and Lean diet groups were analyzed with micro-CT. (b) Threedimensional reconstructions of knee joints were used to visualize calcification of meniscus and osteophyte formation. Gray arrows, ossification of meniscus; white arrows, osteophyte-like structures. Representative 3D images are presented. Bone volume data represent the mean \pm SEM of at least five mice. ${ }^{* *} P<0.01 . n=6$ to 8 mice in each group. degeneration and loss, more-aggressive osteophyte formation in MLI-injured knees from HF-diet mice is associated with a diabetic metabolic phenotype but does not correlate with degree of weight gain.

\section{Discussion}

The results of this study demonstrate that consumption of a high-fat diet that leads to weight gain and diabetes will accelerate the progression of OA that is initiated by induction of a meniscal ligamentous injury. This effect on OA does not correlate, however, with degree of increase in body mass or exposure to a high-fat diet before injury. Our study draws different conclusions from the early work of Silberberg and Silberberg [31,32] who established a link between high-fat diets and OA in rodent models more than 50 years ago. In their pioneering work, they documented an increased incidence of $\mathrm{OA}$ in aged mice on an HF diet with age end points of 12 to 24 months [33]. Interestingly, a conclusion drawn from their data was that the incidence but not the rate of progression of OA was affected by the HF diet [9]. In our current study, we clearly establish that HF diets can accelerate the progression of OA after a defined OA initiation in a diarthrodial joint. Perhaps the variable time of spontaneous initiation, the long timeline of OA progression in the absence of a severe initiating injury, and the absence of quantitative end points may have contributed to the inability of Silberberg and Silberberg to detect changes in rate of progression in their studies. Alternatively, the effects of the HF diet on mechanisms of $\mathrm{OA}$ initiation and progression in aging versus acute injury may be different.

The relative contributions to OA of biomechanical factors resulting from increased body mass versus metabolic dysfunction in the obese and type 2 diabetic population have not been resolved. In humans, a strong correlation has been shown between elevated body mass index (BMI) and OA [21-23]. Biomechanical factors related to this increased body mass have been argued to make critical contributions to OA of the knee $[34,35]$. As for metabolic factors, correlations exist between diabetic parameters (hyperglycemia, hyperinsulinemia) and OA [36-39]. Another strong argument for systemic metabolic factors contributing to $\mathrm{OA}$ in the obese and diabetic population comes from the increased incidence of OA in the hand, a site not susceptible to the biomechanical overload present in the weight-bearing joints of an obese individual $[37,38]$. Logically, detrimental effects of body mass on OA would be a function of both magnitude of body-mass increase and length of exposure to this condition after initiation of OA. Our short-term HF diet group weighed the same as lean controls at the time of injury and gained approximately $6 \mathrm{~g}$ over the following 3-month period. In contrast, the long-term 


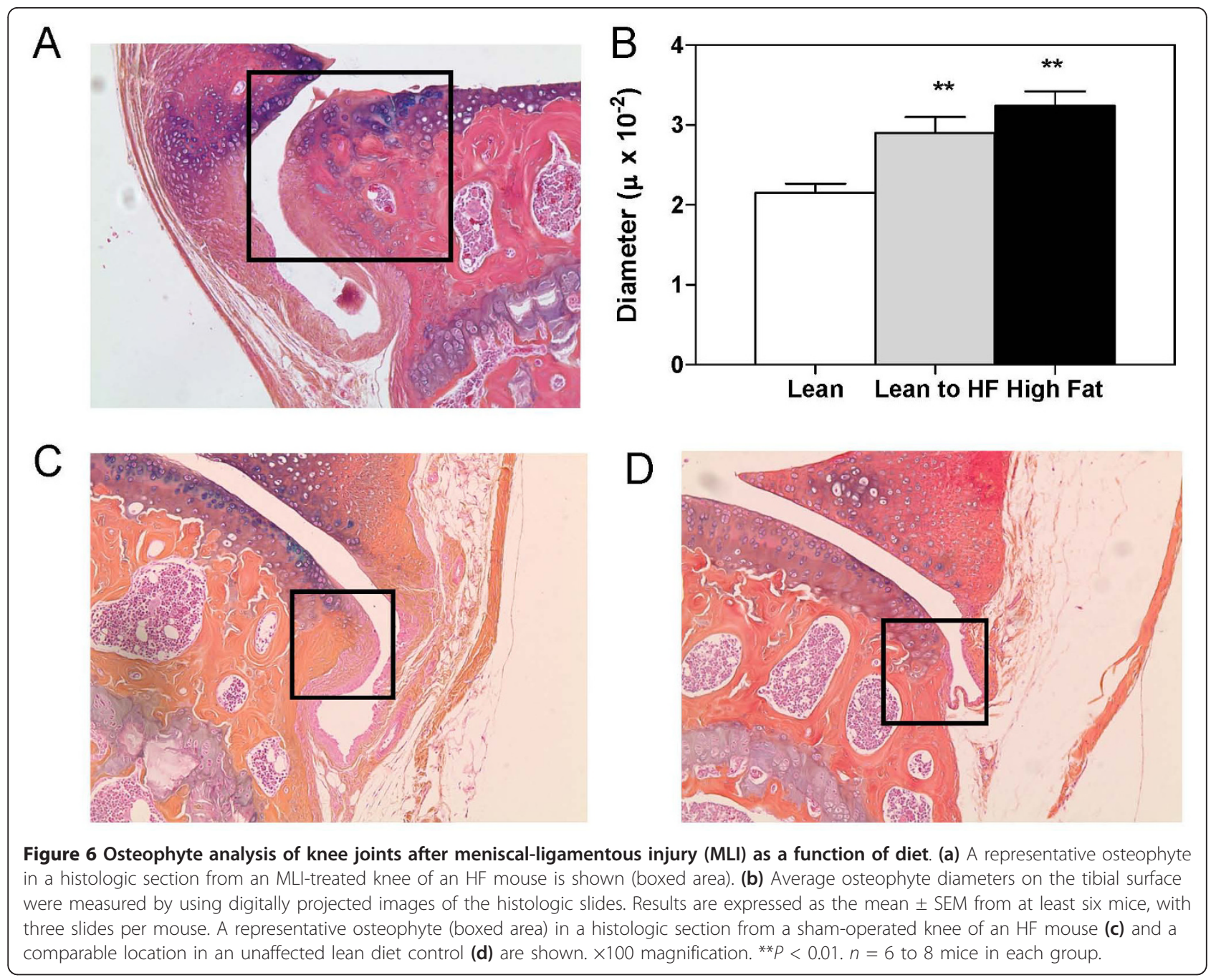

HF-diet group weighed more than $11 \mathrm{~g}$ more than controls at the time of knee injury and increased the differential to more than $20 \mathrm{~g}$ by 3 months. Despite this obvious difference in duration and degree of exposure to increased body mass, the two diet groups had comparable acceleration of OA progression. From our results, we conclude that weight gain does not correlate with progression of OA in our HF-diet mouse model. Silberberg and Silberberg [40] came to a similar conclusion that increased body weight in response to HF diets does not correlate with the incidence of joint disease in mice. Recently, Griffin et al. [41] reported the effect of HF diet-induced obesity on spontaneous OA of aging in female mice at 54 weeks of age and concluded that weight gain and loss of Safranin-O cartilage staining or OA severity score, but not cartilage degeneration, were correlated. Their histologic data suggested a modest OA progression with little loss of cartilage. Perhaps the early stages of $\mathrm{OA}$ initiation and progression are more strongly influenced by body mass. As cartilage degeneration becomes more severe, as in our MLI model, a high-fat diet continues to accelerate progression, and in this phase of the disease, the associated metabolic dysfunction becomes more important.

Silberberg and co-workers [42] did not investigate the metabolic dysfunction in their mouse models as a result of HF diets, but they did observe that the C57BL/ 6 strain but not the DBA strain was susceptible to increased OA of aging when on HF diets, despite weight gain in both strains. Sokoloff et al. [43,44] also observed that OA in the DBA strain was not altered by diet, despite weight gain. Recently, Griffin et al. [45] reported that markedly obese leptin-deficient mice do not have an increased incidence of OA. These observations provide further evidence that factors other than increased body mass are critical to the changes in OA in C57BL/6 mice. Interestingly, C57BL/6 mice are well known for their susceptibility to diabetic changes (hyperinsulinemia, hyperglycemia) when placed on HF diets, whereas DBA mice are resistant to these metabolic changes 
$[29,46]$. Although no cause and effect has been established, these observations show an association between development of a diabetic phenotype and accelerated progression of OA in the C57BL/6 mouse model.

In the mouse, metabolic changes in response to $\mathrm{HF}$ diets appear to be important in mediating osteoarthritic pathogenesis. Saturated fat diets derived from animal fat (lard) are most effective in mediating OA changes $[31,44]$. They are also more effective than dietary sugar in generating obesity and diabetes in experimental mice [47]. N-3 polyunsaturated fat (PUFA), such as linolenic acid, decreases the detrimental effects of the lard diet [48] and decrease expression and activity of proteoglycan-degrading enzymes and expression of inflammatory cytokines in articular chondrocyte cultures [49,50]. As a potential mechanism of action, the n-3 or omega- 3 PUFAs that are found in fish oils have been shown to have anti-inflammatory effects with multiple mechanisms of action being proposed [51]. Importantly, one PUFA, eicosapentaenoic acid, has recently been shown to prevent and reverse insulin resistance in mice fed an HF diet [52]. The mechanism of this latter effect has been proposed to be suppression of adipose tissue inflammation [52]. These results suggest that HF diets mediate their effects on both OA and the diabetic phenotype, at least in part through inflammatory effects.

In relation to $\mathrm{OA}$, the observation that obesity is a proinflammatory state may have critical implications for explaining both the increased progression of $\mathrm{OA}$ in our mouse model of obesity/type 2 diabetes and the epidemiologic data linking obesity to OA. The proinflammatory state of obesity may synergize with other OApromoting pathways to accelerate the progression of the cartilage destruction. Thus, our laboratory is currently investigating the contribution of systemic inflammation to OA progression in obesity as a step toward elucidating novel candidate mechanisms that underlie the deleterious effect of the HF diet on the degenerative process.

\section{Conclusions}

The results of this study indicate that an HF diet accelerates progression of $\mathrm{OA}$ in the $\mathrm{C} 57 \mathrm{BL} / 6$ mouse diabetic model after a meniscal ligamentous injury, as assessed by both cartilage degeneration and osteophyte production. Degree of weight gain does not correlate with these changes, and prior exposure to the HF diet is not necessary for these effects. This suggests that metabolic dysregulation in type 2 diabetes is a comorbid factor in the progression of osteoarthritis, and its effect is in addition to that of obesity-related increased weight bearing in afflicted joints.

\footnotetext{
Abbreviations

HF: high fat; HgbA1c: hemoglobin A1c; JNK: c-Jun N-terminal kinase; kcal: kilocalories; micro-CT: microcomputerized tomography; MLI: meniscal-
}

ligamentous injury; OA: osteoarthritis; OARSI: Osteoarthritis Research Society International; PUFA: polyunsaturated fatty acid.

\section{Acknowledgements}

The authors thank Ryan Tiernay, Nanette Alcock, and Sarah Mack for processing and sectioning of tissue samples; and Michael Thullen for performing micro-CT analyses. This work was supported by an Arthritis Investigator Award from the Arthritis Foundation (MJZ), NIH R01 AR045700 (RNR), NIH P50 AR 054041 (RNR), and the Department of Pathology and Laboratory Medicine, University of Rochester Medical Center (RAM).

\section{Author details}

${ }^{1}$ Department of Pathology and Laboratory Medicine, Box 626, University of Rochester Medical Center, 601 Elmwood Avenue, Rochester, NY 14642, USA. ${ }^{2}$ Center for Musculoskeletal Research, Box 665, University of Rochester Medical Center, 601 Elmwood Avenue, Rochester, NY 14642, USA.

\section{Authors' contributions}

RAM and MJZ conceived the study. RAM participated in the design and coordination of the experiments, carried out the animal studies, and drafted the manuscript. ERS performed the animal surgeries, participated in the scoring of histology slides and the coordination of the study, and helped draft the manuscript. JL performed the tissue processing, histology, histomorphometry, and data analysis. RNR participated in the design and data analysis. MJZ participated in the design and coordination of the experiments, participated in histologic scoring, and helped draft the manuscript. All authors read and approved the final manuscript.

\section{Competing interests}

The authors declare that they have no competing interests.

Received: 2 August 2011 Revised: 26 October 2011

Accepted: 6 December 2011 Published: 7 December 2011

\section{References}

1. Zimmet $P$, Alberti KG, Shaw J: Global and societal implications of the diabetes epidemic. Nature 2001, 414:782-787.

2. Boyle JP, Thompson TJ, Gregg EW, Barker LE, Williamson DF: Projection of the year 2050 burden of diabetes in the US adult population: dynamic modeling of incidence, mortality, and prediabetes prevalence. Popul Health Metr 2010, 8:29.

3. Huang ES, Basu A, O'Grady M, Capretta JC: Projecting the future diabetes population size and related costs for the U.S. Diabetes Care 2009, 32:2225-2229.

4. Arthritis as a potential barrier to physical activity among adults with diabetes: United States, 2005 and 2007. MMWR Morb Mortal Wkly Rep 2008, 57:486-489.

5. Dunbar MJ, Howard A, Bogoch ER, Parvizi J, Kreder HJ: Orthopaedics in 2020: predictors of musculoskeletal need. J Bone Joint Surg Am 2009, 91:2276-2286.

6. Fehring TK, Odum SM, Griffin WL, Mason JB, McCoy TH: The obesity epidemic: its effect on total joint arthroplasty. J Arthroplasty 2007, 22:71-76.

7. Bourne R, Mukhi S, Zhu N, Keresteci M, Marin M: Role of obesity on the risk for total hip or knee arthroplasty. Clin Orthop Relat Res 2007, 465:185-188

8. Guilak F, Fermor B, Keefe FJ, Kraus VB, Olson SA, Pisetsky DS, Setton LA, Weinberg JB: The role of biomechanics and inflammation in cartilage injury and repair. Clin Orthop Relat Res 2004, 423:17-26.

9. Griffin TM, Guilak F: Why is obesity associated with osteoarthritis? Insights from mouse models of obesity. Biorheology 2008, 45:387-398.

10. Sandell L: Obesity and osteoarthritis: is leptin the link? Arthritis Rheum 2009, 60:2858-2860.

11. Xu H, Barnes GT, Yang Q, Tan G, Yang D, Chou CJ, Sole J, Nichols A, Ross JS, Tartaglia LA, Chen H: Chronic inflammation in fat plays a crucial role in the development of obesity-related insulin resistance. J Clin Invest 2003, 112:1821-1830.

12. Weisberg SP, McCann D, Desai M, Rosenbaum M, Leibel RL, Ferrante AW Jr: Obesity is associated with macrophage accumulation in adipose tissue. $J$ Clin Invest 2003, 112:1796-1808. 
13. Lumeng CN, Bodzin JL, Saltiel AR: Obesity induces a phenotypic switch in adipose tissue macrophage polarization. J Clin Invest 2007, 117:175-184.

14. Bastard JP, Maachi M, Van Nhieu JT, Jardel C, Bruckert E, Grimaldi A, Robert JJ, Capeau J, Hainque B: Adipose tissue IL-6 content correlates with resistance to insulin activation of glucose uptake both in vivo and in vitro. J Clin Endocrinol Metab 2002, 87:2084-2089.

15. Hotamisligil GS, Shargill NS, Spiegelman BM: Adipose expression of tumor necrosis factor-alpha: direct role in obesity-linked insulin resistance. Science 1993, 259:87-91.

16. Boden G, She P, Mozzoli M, Cheung P, Gumireddy K, Reddy P, Xiang X, Luo $Z$, Ruderman N: Free fatty acids produce insulin resistance and activate the proinflammatory nuclear factor-kappaB pathway in rat liver. Diabetes 2005, 54:3458-3465.

17. Hamilton JS, Powell LA, McMaster C, McMaster D, Trimble ER: Interaction of glucose and long chain fatty acids (C18) on antioxidant defences and free radical damage in porcine vascular smooth muscle cells in vitro. Diabetologia 2003, 46:106-114.

18. Schrauwen P, Hesselink MK: Oxidative capacity, lipotoxicity, and mitochondrial damage in type 2 diabetes. Diabetes 2004, 53:1412-1417.

19. Evans IL, Goldfine ID, Maddux BA, Grodsky GM: Are oxidative stressactivated signaling pathways mediators of insulin resistance and betacell dysfunction? Diabetes 2003, 52:1-8.

20. Henriksen EJ, Diamond-Stanic MK, Marchionne EM: Oxidative stress and the etiology of insulin resistance and type 2 diabetes. Free Radic Biol Med 2011, 51:993-999.

21. Cicuttini FM, Baker JR, Spector TD: The association of obesity with osteoarthritis of the hand and knee in women: a twin study. J Rheumatol 1996, 23:1221-1226.

22. Lohmander LS, Gerhardsson de Verdier M, Rollof J, Nilsson PM, Engstrom G Incidence of severe knee and hip osteoarthritis in relation to different measures of body mass: a population-based prospective cohort study. Ann Rheum Dis 2009, 68:490-496.

23. Oliveria SA, Felson DT, Cirillo PA, Reed Jl, Walker AM: Body weight, body mass index, and incident symptomatic osteoarthritis of the hand, hip, and knee. Epidemiology 1999, 10:161-166.

24. Kamekura S, Hoshi K, Shimoaka T, Chung U, Chikuda H, Yamada T, Uchida M, Ogata N, Seichi A, Nakamura K, Kawaguchi H: Osteoarthritis development in novel experimental mouse models induced by knee joint instability. Osteoarthritis Cartilage 2005, 13:632-641.

25. Sampson ER, Beck CA, Ketz J, Canary KL, Hilton MJ, Awad H, Schwarz EM, Chen D, O'Keefe RJ, Rosier RN, Zuscik MJ: Establishment of an index with increased sensitivity for assessing murine arthritis. J Orthop Res 2011, 29:1145-1151.

26. Glasson SS, Blanchet TJ, Morris EA: The surgical destabilization of the medial meniscus (DMM) model of osteoarthritis in the 129/SvEv mouse. Osteoarthritis Cartilage 2007, 15:1061-1069.

27. Glasson SS, Chambers MG, Van Den Berg WB, Little CB: The OARSI histopathology initiative: recommendations for histological assessments of osteoarthritis in the mouse. Osteoarthritis Cartilage 2010, 18(Suppl 3): S17-23.

28. Wu Q, Kim KO, Sampson ER, Chen D, Awad H, O'Brien T, Puzas JE, Drissi H, Schwarz EM, O'Keefe RJ, Zuscik MJ, Rosier RN: Induction of an osteoarthritis-like phenotype and degradation of phosphorylated Smad3 by Smurf2 in transgenic mice. Arthritis Rheum 2008, 58:3132-3144.

29. Surwit RS, Kuhn CM, Cochrane C, McCubbin JA, Feinglos MN: Diet-induced type II diabetes in C57BL/6J mice. Diabetes 1988, 37:1163-1167.

30. Nathan DM, Kuenen J, Borg R, Zheng H, Schoenfeld D, Heine RJ: Translating the $\mathrm{A} 1 \mathrm{C}$ assay into estimated average glucose values. Diabetes Care 2008, 31:1473-1478.

31. Silberberg M, Silberberg R: Osteoarthrosis in mice fed diets enriched with animal or vegetable fat. Arch Pathol 1960, 70:385-390.

32. Silberberg $\mathrm{R}$, Silberberg $\mathrm{M}$ : Skeletal growth and articular changes in mice receiving a high-fat diet. Am J Pathol 1950, 26:113-131, incl 114 pl.

33. Silberberg $M$, Silberberg R: Degenerative joint disease in mice fed a highfat diet at various ages. Exp Med Surg 1952, 10:76-87.

34. Sharma L, Song J, Felson DT, Cahue S, Shamiyeh E, Dunlop DD: The role of knee alignment in disease progression and functional decline in knee osteoarthritis. JAMA 2001, 286:188-195.

35. Felson DT, Goggins J, Niu J, Zhang Y, Hunter DJ: The effect of body weight on progression of knee osteoarthritis is dependent on alignment. Arthritis Rheum 2004, 50:3904-3909.
36. Silveri F, Brecciaroli D, Argentati F, Cervini C: Serum levels of insulin in overweight patients with osteoarthritis of the knee. J Rheumatol 1994, 21:1899-1902.

37. Dahaghin S, Bierma-Zeinstra SM, Koes BW, Hazes JM, Pols HA: Do metabolic factors add to the effect of overweight on hand osteoarthritis? The Rotterdam Study. Ann Rheum Dis 2007, 66:916-920.

38. Douloumpakas I, Pyrpasopoulou A, Triantafyllou A, Sampanis C, Aslanidis S: Prevalence of musculoskeletal disorders in patients with type 2 diabetes mellitus: a pilot study. Hippokratia 2007, 11:216-218

39. Hart DJ, Doyle DV, Spector TD: Association between metabolic factors and knee osteoarthritis in women: the Chingford Study. J Rheumatol 1995, 22:1118-1123.

40. Silberberg M: Effects of a high fat diet on the joints of aging mice. Arch Pathol 1950, 50:828-846.

41. Griffin TM, Fermor B, Huebner JL, Kraus VB, Rodriguiz RM, Wetsel WC, Cao L, Setton $L A$, Guilak F: Diet-induced obesity differentially regulates behavioral, biomechanical, and molecular risk factors for osteoarthritis in mice. Arthritis Res Ther 2010, 12:R130.

42. Silberberg M, Silberberg R: Role of thyroid hormone in the pathogenesis of joint disease in mice: effects of radiothyroidectomy and high-fat diets. J Bone Joint Surg Am 1955, 37-A:537-548.

43. Sokoloff L, Mickelsen O, Silverstein E, Jay GE Jr, Yamamoto RS: Experimental obesity and osteoarthritis. Am J Physiol 1960, 198:765-770.

44. Sokoloff $L$, Mickelsen O: Dietary fat supplements, body weight and osteoarthritis in Dba-2jn mice. J Nutr 1965, 85:117-121.

45. Griffin TM, Huebner JL, Kraus VB, Guilak F: Extreme obesity due to impaired leptin signaling in mice does not cause knee osteoarthritis. Arthritis Rheum 2009, 60:2935-2944.

46. Surwit RS, Seldin MF, Kuhn CM, Cochrane C, Feinglos MN: Control of expression of insulin resistance and hyperglycemia by different genetic factors in diabetic C57BL/6J mice. Diabetes 1991, 40:82-87.

47. Surwit RS, Feinglos MN, Rodin J, Sutherland A, Petro AE, Opara EC, Kuhn CM, Rebuffe-Scrive M: Differential effects of fat and sucrose on the development of obesity and diabetes in C57BL/6J and A/J mice. Metabolism 1995, 44:645-651.

48. Silberberg M, Silberberg R, Orcutt B: Modifying effect of linoleic acid on articular aging and osteoarthrosis in lard-fed mice. Gerontologia 1965, 11:179-187.

49. Curtis $C L$, Hughes $C E$, Flannery $C R$, Little CB, Harwood JL, Caterson B: $n-3$ fatty acids specifically modulate catabolic factors involved in articular cartilage degradation. J Biol Chem 2000, 275:721-724.

50. Curtis $C L$, Rees $S G$, Cramp J, Flannery CR, Hughes $C E$, Little $C B$, Williams $R$, Wilson C, Dent CM, Harwood JL, Caterson B: Effects of $n-3$ fatty acids on cartilage metabolism. Proc Nutr Soc 2002, 61:381-389.

51. Chapkin RS, Kim W, Lupton JR, McMurray DN: Dietary docosahexaenoic and eicosapentaenoic acid: emerging mediators of inflammation. Prostaglandins Leukot Essent Fatty Acids 2009, 81:187-191.

52. Kalupahana NS, Claycombe K, Newman SJ, Stewart T, Siriwardhana N, Matthan N, Lichtenstein AH, Moustaid-Moussa N: Eicosapentaenoic acid prevents and reverses insulin resistance in high-fat diet-induced obese mice via modulation of adipose tissue inflammation. J Nutr 2010, 140:1915-1922

\section{doi:10.1186/ar3529}

Cite this article as: Mooney et al:: High-fat diet accelerates progression of osteoarthritis after meniscal/ligamentous injury. Arthritis Research \& Therapy 2011 13:R198. 